\section{Themenkomplex VI: Barrett-Ösophagus}

H. Messmann, C. Ell, M. Fein, R. Kiesslich, M. Ortner, R. Porschen, M. Stolte

\section{Definition des Barrett-Ösophagus \\ Konsens}

Die Diagnose des Barrett-Ösophagus (BÖ) wird histologisch gestellt durch Nachweis von intestinalisiertem metaplastischen Zylinderepithel. Abhängig von der endoskopisch nachweisbaren Länge der Zylinderepithelmetaplasie im distalen Ösophagus unterscheidet man einen Long-Segment-Barrett-Ösophagus (LSB) $(\geq 3 \mathrm{~cm})$ und einen Short-Segment-Barrett-Ösophagus (SSB) $(<3 \mathrm{~cm})(\mathrm{C})$.

\section{Kommentar}

Es bestand Konsens, dass zur Diagnosestellung eines BÖ der histologische Nachweis von intestinalisiertem metaplastischen $\mathrm{Zy}$ linderepithel (intestinale Metaplasie Typ III) erforderlich und gleichzeitig die endoskopische Längenangabe unverzichtbar ist $[1,2]$. Dennoch können aufgrund des fokalen Auftretens von spezialisiertem Barrett-Epithel der endoskopische Verdachtsbefund und die histologische Bestätigung aufgrund von „sampling errors“ differieren [3]. Die willkürlich gewählte Länge von $\geq 3 \mathrm{~cm}$ für den LSB und $\tau 3 \mathrm{~cm}$ für den SSB wird beibehalten.

Der mikroskopische Barrett-Ösophagus ist definiert als histopathologischer Nachweis von spezialisiertem Epithel bei unauffälliger Z-Linie. Davon abzugrenzen ist die intestinale Metaplasie der Kardiaschleimhaut, die mit einer H.-p.-Gastritis assoziiert ist und meist eine komplette intestinale Metaplasie (Typ I) darstellt [4].

\section{Diagnose des Bö \\ Konsens}

Die Bestimmung des gastroösophagealen Überganges erfolgt endoskopisch und entspricht dem proximalen Ende der Magenfalten ohne Luftinsufflation und ohne Peristaltik (C).

\section{Kommentar}

Die Bestimmung des gastroösophagealen Überganges erfolgt endoskopisch. Die proximalen Magenfalten bestimmen dabei den gastroösophagealen Übergang. Dennoch ergeben sich Schwierigkeiten bei starker Peristaltik, schlecht sedierten Patienten oder bei großen axialen Hiatushernien [5].

Bei Patienten mit GERD findet sich signifikant häufiger ein BÖ, weswegen diese Patienten zum Nachweis oder Ausschluss eines BÖ zumindest einmal in ihrer Krankengeschichte endoskopiert 
werden sollten. Lieberman fand ein 10fach höheres Risiko für einen Barrett-Ösophagus, wenn eine GERD mehr als 10 Jahre vorlag im Vergleich zu einer einjährigen Beschwerdesymptomatik [6-9].

Da bereits geringgradige Veränderungen der Schleimhaut (Rötungen, „Zungen“) Ausdruck eines BÖ, einer Präkanzerose i. S. von intraepithelialen Neoplasien (IEN) oder ein Frühkarzinom sein können, sind Videoendoskope mit hoher Auflösung zur Erkennung dieser Läsionen empfehlenswert.

\section{Konsens}

Um einen BÖ zu diagnostizieren, sind Quadrantenbiopsien alle $1-2 \mathrm{~cm}$ innerhalb der vermuteten Barrett-Schleimhaut notwen$\operatorname{dig}(B)$.

Besteht der V.a. eine geringgradige intraepitheliale Neoplasie (IEN) in Kombination mit histologisch oder endoskopisch nachweisbaren regeneratorischen Veränderungen, sollte nach einer 4- bis 6-wöchigen Protonenpumpenhemmer(PPI)-Therapie eine Kontrollendoskopie mit nochmaliger Biopsie erfolgen (C).

Die (Magnifikations-)Chromoendoskopie mit Methylenblau (MB) hat derzeit noch keinen Stellenwert für die Diagnosestellung des BÖ (B).

\section{Kommentar}

Die Anzahl der zu entnehmenden Biopsien, um einen BÖ initial zu diagnostizieren, ist bislang nicht definiert $[1,10,11]$. Die Interobserver-Variation in der Diagnostik von IEN, insbesondere wenn es um die Beurteilung von geringgradigen IEN in regeneratorisch verändertem Gewebe geht, ist beträchtlich. Deswegen wird die Zweitmeinung eines auf diesem Gebiet erfahrenen Pathologen empfohlen [12 - 14]. Um die Beurteilung möglichst unter optimalen Bedingungen durchzuführen, sollte eine konsequente Therapie mit einem PPI für ca. 4-6 Wochen durchgeführt werden.

Die Chromoendoskopie mit Methylenblau zeigte teils kontroverse Daten in der Detektion von intestinalisiertem Barrett-Epithel [15-20].

\section{Konsens}

Eine Biopsie im Bereich des gastroösophagealen Übergangs bei unauffälliger Z-Linie bei Patienten mit Refluxbeschwerden ist nicht erforderlich (C). Bei einer Biopsie zur Diagnose des BÖ sollen gleichzeitig je zwei PEs aus Antrum und Korpus zur Evaluation einer Helicobacter-pylori(HP)-induzierten Gastritis entnommen werden (B).

\section{Kommentar}

Der Nachweis eines mikroskopischen BÖ hat derzeit keine klinische Relevanz, d.h., es ergibt sich daraus weder die Konsequenz einer Überwachung noch einer Therapie.

Der HP-Status ist in einigen Fällen hilfreich zur Differenzierung zwischen SSB und intestinaler Metaplasie der Kardia, da sich HP signifikant weniger beim SSB fand als im Vergleich zur intestinalen Metaplasie der Kardia [21 -24]. Außerdem ist bei einer PPIDauertherapie eine HP-Eradikation wegen der Verstärkung der Aktivität einer HP-Korpusgastritis zu diskutieren [25, 26] (s. u.).

\section{Konsens}

Bei histologisch gesichertem BÖ sollten zum Screening von IEN zunächst alle makroskopisch suspekten Areale biopsiert werden. Anschließend sollte die Quadrantenbiopsie im Abstand von $1-2 \mathrm{~cm}$ mit einer Standardzange durchgeführt werden.

Färbe- und Fluoreszenzverfahren zur Dysplasie-Detektion können derzeit für die Routine nicht empfohlen werden. Die Bürstenzytologie hat keinen Stellenwert (B).

Die Durchflusszytometrie kann bei lokaler Verfügbarkeit eine sinnvolle Ergänzung darstellen (B).

\section{Kommentar}

Vor der Quadrantenbiopsie sollten makroskopisch suspekte Läsionen gezielt biopsiert werden. Biopsien in $1 \mathrm{~cm}$ Abstand in Analogie zum Seattle-Protokoll sind schwer praktikabel [27], umgekehrt besteht bei $\geq 2 \mathrm{~cm}$ Abstand das Risiko, dass bis zu 50\% der Karzinome nicht erfasst werden [28]. Die Bürstenzytologie erlaubt keinen sicheren Nachweis von IEN $[29,30]$.

Die Färbung mit Methylenblau zur gezielten Detektion von IEN liefert teils kontroverse Daten $[15,17]$. Zur Fluoreszenzendoskopie liegen bislang keine randomisierten Studien vor. Das Verfahren hat noch experimentellen Charakter [31].

Reid [32] und Teodori [33] zeigten in Langzeitbeobachtungen (> 13 Jahre) bei Patienten mit BÖ, dass es mittels Flow-Zytometrie möglich ist, Risikopatienten zu definieren, die gehäuft eine IEN oder ein Karzinom zu entwickeln.

\section{Endoskopischer Ultraschall (EUS) \\ Konsens}

Der EUS hat zur initialen Diagnosestellung eines BÖ keinen Stellenwert. Beim histologischen Nachweis eines Karzinoms sollte zum Staging eine Endosonographie durchgeführt werden, wenn sich daraus eine klinische Relevanz ergibt. Beim Nachweis von IEN ist der Stellenwert des EUS nicht gesichert (C).

\section{Kommentar}

Bei der initialen Diagnosestellung des BÖ hat der EUS keinen Stellenwert. Der EUS mit 7,5 MHz eignet sich nicht, IEN von Frühkarzinomen zu unterscheiden, da eine sichere Differenzierung von hochgradigen IEN und Karzinomen nicht möglich war [34]. Hochauflösende Ultraschallminisonden $(\geq 20 \mathrm{MHz}$ ) tragen bei mukosalen Karzinomen möglicherweise zu einem besseren lokalen Staging der T-Kategorie bei [35]. Bei der Frage nach pathologisch vergrößerten LK bleibt die EUS (7,5 MHz) jedoch die Methode der Wahl [36].

\section{Indikatoren bzw. Risikofaktoren der Adenokarzinomentwicklung Konsens}

Der Nachweis einer IEN, ein langer BÖ, die Präsenz eines Ulkus sowie sichtbare Mukosaerhabenheiten stellen histologische bzw. endoskopische Indikatoren für ein Barrett-Adenokarzinom dar (B). 


\section{Kommentar}

Der Nachweis einer IEN ist derzeit der einzige histologische Risikofaktor für die Entwicklung eines Barrett-Adenokarzinoms [37, 38]. Neben der Präsenz von IEN zum Diagnosezeitpunkt ist der Schweregrad der IEN für den weiteren Verlauf entscheidend [37].

Sampliner wertete 4 Studien retrospektiv aus: Liegt keine IEN vor, traten im Zeitraum von 2,4-10 Jahren in 3\% Karzinome auf. Lagen geringgradige bzw. hochgradige IEN vor, stieg das Karzinomrisiko auf 18 bzw. 34\% im Zeitraum von 1,5-4,3 bzw. 0,2-4,3 Jahren.

Der endoskopische Nachweis eines sehr langen Barrett-Ösophagus $(\tau 8-10 \mathrm{~cm})$ wird in der Mehrzahl der Studien als Risikofaktor für ein Barrett-Adenokarzinom gesehen [37, 39-41]. Das Barrett-Ulkus und die sichtbare „Nodularität“ sind weitere endoskopische Risikofaktoren [39, 42, 43].

\section{Konsens}

Die Bestätigung bzw. der Nachweis einer IEN sollte durch die Zweitmeinung eines unabhängigen, auf diesem Gebiet sehr erfahrenen und möglichst externen Pathologen erfolgen (B). Eine solche Zweitbegutachtung soll auch für Fälle mit schwieriger Abgrenzung zwischen hochgradigen IEN und einem invasiven Karzinom durchgeführt werden (C).

Bei Nachweis einer DNA-Aneuploidie und/oder einer erhöhten G2/M-Phase kann die Überwachung intensiviert werden (B). Der Nachweis von p53, HER2 u.a. ist Gegenstand klinischer Forschung und muss durch Langzeitbeobachtung besser evaluiert werden (B).

\begin{tabular}{|lll}
\hline IEN-Grad & LSB & SSB \\
\hline keine IEN & $\begin{array}{l}\text { nach 2 neg. Kontrollen im 1. Jahr } \\
\text { alle 3 Jahre }\end{array}$ & $\begin{array}{l}\text { nach 2 neg. Kon- } \\
\text { trollen im 1. Jahr } \\
\text { alle 4 Jahre }\end{array}$ \\
\hline $\begin{array}{l}\text { geringgradige } \\
\text { IEN }\end{array}$ & $\begin{array}{l}\text { im Abstand von 6 Monaten (2-mal), } \\
\text { dann jährlich. Bei Nachweis von ge- } \\
\text { ringgradigen IEN in mukosalen Erha- } \\
\text { benheiten sollte eine endoskopische } \\
\text { Resektion (ER) dieser Läsion ange- } \\
\text { strebt werden. }\end{array}$ & wie LSB \\
\hline hochgradige IEN & $\begin{array}{l}\text { Bei sichtbarer Läsion ER oder bei nicht } \\
\text { sichtbarer hochgradiger IEN PDT, } \\
\text { alternativ Operation }\end{array}$ & \\
\hline
\end{tabular}

\section{Kommentar}

Reid zeigte, dass die Übereinstimmung von Pathologen für die Unterscheidung „hochgradige IEN/Karzinom“ von „geringgradige IEN/unbestimmte IEN/keine IEN“ bei über $87 \%$ liegt, während die Entscheidung IEN ,ja oder nein“ nur 72\% Übereinstimmung fand [13]. Die Empfehlung, bei der Diagnostik von IEN die Zweitmeinung eines Pathologen einzuholen, wurde ebenso in der Arbeitsgemeinschaft Gastroenterologische Pathologie der Deutschen Gesellschaft für Pathologie ausgesprochen [44]. Mehrere Studien zeigen eine erhöhte Expression von p53 und HER2 für hochgradige IEN und Karzinom [45, 46]. Neuere Daten zeigen, dass selbst bei geringgradigen IEN und dem Nachweis von p53 ein erhöhtes Progressionsrisiko zu hochgradigen IEN bzw. Karzinom besteht [47].

\section{Überwachung des BÖ \\ Konsens}

Die Überwachung scheint für alle Patienten sinnvoll, bei denen eine operative oder endoskopische Therapie im Falle der Entdeckung eines Tumors möglich erscheint (B).

\section{Kommentar}

Bislang existieren nur retrospektive Studien, die einen Vorteil der regelmäßigen endoskopischen Überwachung beim BÖ belegen [48-53].

Die Kosten der Überwachung hängen von mehreren Faktoren, insbesondere von der Vergütung im jeweiligen Gesundheitssystem, ab. Soni et al. zeigten in ihrer Studie zur Kosten-NutzenAnalyse des Screenings von Patienten mit Refluxerkrankung, dass im Vergleich zur Nichtdurchführung einer regelmäßigen Vorsorge eine „Screening-Endoskopie“ pro gerettetem Lebensjahr 24700 US-Dollar kostet. In einer englischen Studie wurden die Kosten für ein entdecktes Karzinom mit 15000 (Männer) bzw. 42000 engl. Pfund (Frauen) berechnet [54]. Berechnungen für das deutsche Gesundheitssystem existieren nicht, dürften aber deutlich darunter liegen.

„Gegner“ der Überwachung argumentieren, dass ein Großteil der Patienten mit BÖ nicht am Karzinom, sondern an anderen Zweiterkrankungen stirbt $[39,55]$. Diese Studien unterstreichen noch einmal die Wichtigkeit, nur solche Patienten zu überwachen, bei denen die Entdeckung von IEN eine klinische Konsequenz nach sich zieht.

Obwohl das Barrett-Adenokarzinom in den letzten Jahren deutlich zugenommen hat, bleibt dennoch festzustellen, dass neue Studien die Inzidenz für Barrett-Adenokarzinome etwas geringer ansetzen als zunächst angenommen (entsprechend einer Inzidenz von 1/285 Patientenjahren) [56].

Shaheen et al. zeigten, dass das Karzinomrisiko von Patienten mit BÖ aufgrund eines Publikationsbias überschätzt wird [57]. Obwohl das genaue Karzinomrisiko nicht bekannt ist, vermuten Shaheen et al. nach diesen Ergebnissen, dass eine jährliche Karzinominzidenz von 0,5\% einer realistischen Schätzung entspricht. Unklar ist, ob es in den westlichen Ländern geographische Unterschiede gibt [58]. Trotz des möglicherweise geringen Karzinomrisikos sollten unserer Meinung nach diese Patienten überwacht werden, wobei die Überwachungsintervalle eventuell neu definiert werden sollten. Dass die Überwachungsintervalle in größeren Abständen, z. B. alle 4 Jahre, ausreichend sein können, zeigt die Studie von Provenzale et al. [59].

Basierend auf einem computergestützten Modell untersuchten die Autoren die Wertigkeit unterschiedlicher endoskopischer Überwachungsstrategien für Patienten mit BÖ in Abhängigkeit von der Karzinominzidenz. Für eine jährliche Karzinominzidenzrate von 0,2\% lautet nach diesen Daten die Empfehlung, keine endoskopische Überwachung durchzuführen. Bei einer Inzidenzrate von $1 \%$ bzw. 0,5\% sollte unter Berücksichtigung der Kosten-Nutzen-Analyse eine endoskopische Überwachung im 
Abstand von zwei bzw. vier Jahren erfolgen. Die neuen Empfehlungen von 2002 des „American College of Gastroenterology“ zur Überwachung bzw. Therapie von Patienten mit BÖ werden modifiziert übernommen [60].

\section{Konsens}

Überwachungsintervalle bzw. Therapieempfehlungen bei Patienten mit BÖ in Abhängigkeit vom IEN-Grad und der Länge des BÖ (C):

\section{Kommentar}

Beim Nichtvorhandensein von IEN wird zwischen LSB und SSB unterschieden, da das Karzinomrisiko beim SSB geringer ist als beim LSB [61]. Eine Verlängerung der Überwachungsintervalle beim SSB ohne IEN scheint unter diesem Aspekt gerechtfertigt. Bei Patienten mit geringgradigen IEN in einer sichtbaren Erhabenheit (Nijhawan et al., 2000) empfehlen wir eine diagnostische ER. Das Vorhandensein einer hochgradigen IEN ist in etwa $40 \%$ mit dem Vorhandensein von nicht sichtbaren Karzinomen assoziiert [62]. Außerdem zeigte Weston an 15 Patienten mit unifokalen hochgradigen IEN, dass es im Verlauf von 3 Jahren in 53,3\% zu einem Progress (multifokale hochgradige IEN/Karzinom) kommt [63]. Demgegenüber steht zwar die Arbeit von Schnell et al., die zeigt, dass nach 6 Jahren nur in 15\% Karzinome auftraten und das Vorhandensein eines Karzinoms das Überleben nicht beeinflusste [64]. Zu dieser Arbeit muss jedoch kritisch angemerkt werden, dass bei 738 von 1099 untersuchten Patienten (67\%) mit BÖ eine geringgradige IEN diagnostiziert wurde. Dieser hohe Anteil an geringgradigen IEN in einem Kollektiv von Patienten mit BÖ ist bisher einmalig in der Literatur und lässt Zweifel an der richtigen histopathologischen Diagnose aufkommen.

Treten hochgradige IEN multifokal auf, so ist das Karzinomrisiko zusätzlich erhöht [43]. Ob eine sichere Differenzierung von hochgradigen IEN und Karzinom mittels Biopsietechnik („Seattle-Biopsy-Protocol“) möglich ist, wird kontrovers diskutiert [27, 65]. Aufgrund dieser Unsicherheit und des erhöhten Karzinomrisikos wird die Therapie der hochgradigen IEN in Analogie zum Frühkarzinom empfohlen.

\section{Therapie}

\section{Therapie des BÖ ohne IEN Konsens}

Die Therapie erfolgt in Analogie zu Patienten mit Refluxbeschwerden, d.h., die Entscheidung, ob eine PPI-Therapie oder eine Antirefluxoperation durchgeführt wird, sollte unabhängig von der Diagnose BÖ erfolgen. Asymptomatische Patienten mit BÖ bedürfen keiner speziellen Therapie (B). Eine prophylaktische Ablation der Barrett-Schleimhaut ist nicht indiziert (B).

\section{Kommentar}

Eine Karzinomprophylaxe durch Säuresuppression mit PPI konnte bislang in keiner klinischen Studie gezeigt werden, wenngleich In-vitro-Daten Hinweise liefern, dass sich unter Säuresuppression die Differenzierungs- (Villin) und Proliferationsmarker (PCNA) erhöhen bzw. erniedrigen [66]. Bei bereits nachgewiesenen hochgradigen IEN war eine ungenügende Säuresuppression ein Risikofaktor für die Progression zum Karzinom [43]. Daraus könnte man schlussfolgern, dass eine konsequente PPI-Dauertherapie das Karzinomrisiko reduziert. Es gibt jedoch keine kontrollierten Studien, die diese Rationale belegen.

Eine konsequente Säuresuppression mit PPI führt allenfalls zur Vergrößerung der Plattenepithelinseln und zu einer umschriebenen Restitution durch Plattenepithel, eine völlige Rückbildung des BÖ ist nicht zu erwarten [67-72]. Auch nach chirurgischer Antirefluxoperation ist eine Rückbildung des BÖ nicht zu erwarten, auch das Karzinomrisiko lässt sich nicht reduzieren [73, 74].

Derzeit gibt es keine randomisierten Studien, die einen klinischen Vorteil einer PPI-Dauertherapie bei asymptomatischen Patienten unterstützen.

In zahlreichen Studien wurde der Stellenwert einer Ablation der Barrett-Schleimhaut untersucht. Athermische Verfahren wie die PDT konnten ebenso wie thermische Verfahren (Argonplasmakoagulation, Nd-YAG-Laser, multipolare Elektrokoagulation, KTP-Laser) die Barrett-Schleimhaut abladieren [75-81]. Eine suffiziente Säuresuppression ist Vorraussetzung, dass sich aus den verbliebenen Stammzellen Plattenepithel ausbildet. Mit keinem der Verfahren war es bislang möglich, die Barrett-Schleimhaut komplett zu abladieren, außerdem verbleiben oftmals Barrett-Schleimhautmetaplasien, die als „Zellnester“ unter dem sich neu bildenden Plattenepithel nachzuweisen sind. Eine nachfolgende Überwachung solcher Patienten dürfte somit eher schwieriger werden.

Eine prophylaktische Ablation der Barrett-Schleimhaut ohne IEN zur Karzinomprophylaxe sollte außerhalb von Studien nicht durchgeführt werden.

\section{Therapie des BÖ mit IEN (geringgradig und/oder hochgradig) Konsens}

Bei Patienten mit geringgradigen IEN sind jährliche Kontrollen ausreichend. Treten geringgradige IEN in einer Schleimhautunregelmäßigkeit oder Erhabenheit auf, sollte eine ER dieser Läsion angestrebt werden.

Bei Patienten mit hochgradigen IEN ist eine Therapie indiziert. Neben der Operation stehen mit den endoskopischen Verfahren (PDT, ER) minimalinvasive Verfahren zur Verfügung, die hinsichtlich Effektivität als gleichwertig zur Operation anzusehen sind, aber hinsichtlich Morbidität und Mortalität der Operation Vorteile bieten (B).

\section{Kommentar}

Die Operation war lange Zeit als Goldstandard anzusehen, ist aber mit einer Mortalität von 5-20\% und Morbidität von $30-40 \%$ assoziiert [82, 83]. Neuerdings stehen mit den endoskopischen Verfahren im Ergebnis wohl gleichwertige und im Hinblick auf Komplikationen und Nebenwirkungen wohl bessere Daten als bei der Operation zur Verfügung.

Die PDT mit 5-Aminolävulinsäure konnte in 2 Pilotstudien mit insgesamt jedoch nur 15 Patienten eine 100\%ige Ablation erzielen $[84,85]$, eine randomisierte Studie an 18 weiteren Patienten bestätigte diesen Erfolg [86]. Overholt konnte nach Sensibilisie- 
rung mit Photofrin bei 56 von 73 Patienten mit hochgradigen IEN eine komplette Ablation erreichen [87]. Mittlerweile liegt ein Follow-up von im Mittel 59 Monaten bei 82 von 103 Patienten vor. In $94 \%$ war eine komplette Ablation der hochgradigen IEN möglich, die Karzinominzidenz lag bei $3 \%$ im Vergleich zu einer erwarteten Inzidenz von 25 - 50\% bei unbehandelten Patienten. Als wesentlicher Nachteil wird eine Strikturrate von 18\% nach der 1. Sitzung und insgesamt von $30 \%$ bei mehrfacher PDT angegeben [88].

Mittlerweile ist in den USA Photofrin für die Behandlung der hochgradigen IEN beim Barrett-Ösophagus zugelassen. In der Zulassungsstudie wurden 208 Patienten $(2: 1)$ in einen PDT-Arm $(\mathrm{n}=138)$ und in einen Kontrollarm $(\mathrm{n}=70)$ randomisiert. Es zeigte sich ein hochsignifikanter Therapieerfolg $(p=0,0001)$ in der Eradikation der hochgradigen IEN mittels PDT (77\%) vs. $39 \%$. Die Progression zum Karzinom war im Kontrollarm während des Beobachtungszeitraums von 2-3,6 Jahren doppelt so hoch [89].

Sind die Veränderungen einer hochgradigen IEN makroskopisch sichtbar, ist die ER die Methode der Wahl, zumal dieses Verfahren eine histologische Beurteilung erlaubt [42, 90, 91]. Die Fallzahlen zur Argonplasmakoagulation sind noch geringer und zeigten in einer Studie von Van Laethem in 8 von 10 Patienten eine Ablation von hochgradigen IEN [92]. Aufgrund der Nachteile der Operation (Mortalität, Morbidität) und der Vorteile der endoskopischen Methoden (minimalinvasiv, kostengünstig) ist zu erwarten, dass unter Berücksichtigung vergleichbarer Langzeitergebnisse für beide Verfahren die endoskopische Therapie die Methode der Wahl werden wird. Bei den endoskopischen Verfahren sollte, wo möglich, die ER (sichtbare Läsionen) der PDT vorgezogen werden, da nur so eine genaue histopathologische Beurteilung möglich ist. Bei endoskopisch nicht sichtbaren dysplastischen Arealen kann die PDT eingesetzt werden. Unklar ist, welcher Sensibilisator sich hier etablieren wird. Die Kombination aus zunächst ER gefolgt von PDT scheint viel versprechend [93].

Von Seiten der Chirurgie sind neue, weniger invasive Operationsverfahren mit niedriger Mortalität und Morbidität propagiert worden [94, 95]. Hier liegen noch keine Langzeitüberlebensdaten vor, daher ist auch im Vergleich zur PDT und ER noch keine endgültige Wertung möglich.

\section{Therapie des Barrett-Adenofrühkarzinoms (mukosales und die Submukosa infiltrierendes Karzinom) Konsens}

Neben der Operation als etablierte Methode kann die ER oder die PDT als alternative Therapie beim intramukosalen Karzinom eingesetzt werden.

Bei Infiltration der Submukosa ist die Operation die Methode der Wahl.

Die Morbidität und Mortalität der endoskopischen Therapieverfahren sind niedriger als bei der operativen Methode (B).

\section{Kommentar}

Ell et al. führten bei 64 Patienten mit Frühkarzinomen/hochgradigen IEN eine ER durch. Eine komplette Remission wurde bei „low-risk“-Fällen ( $<20 \mathrm{~mm}$ bzw. Typ I, Ila, b, c $<10 \mathrm{~mm}$, G1/2, mukosal) in $97 \%$, bei „high-risk“-Fällen in 59\% erzielt. Das Follow-up betrug $12 \pm 8$ Monate und die Rezidivrate lag bei $14 \%$ (Ell et al., 2000). Dieses Verfahren stellt derzeit insbesondere bei multimorbiden Patienten mit erhöhtem OP-Risiko das Verfahren der Wahl dar. Vorliegende Langzeitdaten zu dieser Technik bestätigen die gleiche Effektivität wie die Operation bei geringerer Morbidität und Mortalität [91, 96]. Alternativ kann bei schlecht abgrenzbarem Karzinom oder gleichzeitigem Vorliegen von hochgradigen IEN eine PDT durchgeführt werden. Für Photofrin als Sensibilisator lagen die Ansprechraten bei T1- bzw. T2-Tumoren bei 77-87\% nach 5 Jahren [97]. Die die Submukosa infiltrierende Karzinome sollten primär nicht endoskopisch therapiert werden, da in $20 \%$ bereits Lymphknotenmetastasen vorliegen können. Wird 5-Aminolävulinsäure eingesetzt, konnte Gossner für T1-Tumoren in 77\% eine komplette Remission erzielen [85]. Nachteile der PDT mit Photofrin als Sensibilisator sind eine über mehrere Wochen anhaltende Lichtempfindlichkeit und die Gefahr einer Striktur in bis zu 30\%.

\section{Nachsorge beim endoskopisch therapierten Barrett-Adenokarzinom/IEN Konsens}

Nach erfolgreicher endoskopischer Therapie von hochgradigen IEN/Karzinom sollten folgende Kontrollintervalle eingehalten werden: vierteljährlich im 1. Jahr, halbjährlich im 2. Jahr, dann jährlich bis zum 5. Jahr (C).

\section{Kommentar}

Es gibt bislang keine standardisierten Nachsorgeprotokolle. Die empfohlenen Zeitintervalle sind willkürlich festgelegt.

\section{Literatur}

${ }^{1}$ Sampliner RE. Practice guidelines on the diagnosis, surveillance, and therapy of Barrett's esophagus. Am J Gastroenterol 1998; 93: $1028-1032$

${ }^{2}$ Sharma P, Morales TG, Sampliner RE. Short segment Barrett's esophagus - the need for standardization of the definition and of endoscopic criteria. Am J Gastroenterol 1998; 93: 1033 - 1036

${ }^{3}$ Eloubeidi MA, Provenzeale D. Does this patient have Barrett's esophagus? The utility of predicting Barrett's esophagus at the index endoscopy. Am J Gastroenterol 1999; 94: 937-943

${ }^{4}$ Glickman JN, Wang H, Das KM et al. Phenotype of Barrett's esophagus and intetinal metaplasia of distal esophagus and gastroesophageal junction. Am J Surg Pathol 2001; 25: 87-94

5 McClave SA, Boyce HW Jr, Gottfried MR. Early diagnosis of columnarlined esophagus: a new endoscopic diagnostic criterion. Gastrointest Endosc 1987; 33: 413-416

${ }^{6}$ de Mas CR, Kramer M, Seifert E et al. Short Barrett: prevalence and risk factors. Scand J Gastroenterol 1999; 34: 1065 - 1070

${ }^{7}$ Eisen GM, Sandler RS, Murray S et al. The relationship between gastroesophageal reflux disease and its complications with Barrett's esophagus. Am J Gastroenterol 1997; 92: 27-31

${ }^{8}$ Freston JW, Malagelada JR, Petersen $\mathrm{H}$ et al. Critical issues in the management of gastroesophageal reflux disease. Eur J Gasteroenterol Hepatol 1995; 7: 577-586

${ }^{9}$ Lieberman DA, Oehlke M, Helfand M. Risk factors for Barrett's esophagus in community-based practice. GORGE consortium. Gastroenterology Outcomes Research Group in Endoscopy. Am J Gastroenterol 1997; 92: $1293-1297$ 
${ }^{10}$ Canto MI. Staining in gastrointestinal endoscopy: the basics. Endoscopy 1999; 31: 479-486

11 Weinstein WM, Ippoliti AF. The diagnosis of Barrett's esophagus: goblets, goblets, goblets. Gastrointest Endosc 1996; 44: 91 - 95

12 Alikhan M, Rex D, Khan A et al. Variable pathologic interpretation of columnar lined esophagus by general pathologists in community practice. Gastrointest Endosc 1999; 50: 23 -26

13 Reid BJ, Haggitt RC, Rubin CE et al. Observer variation in the diagnosis of dysplasia in Barrett's esophagus. Hum Pathol 1988; 19: 166- 178

${ }^{14}$ Skacel M, Petras RE, Gramlich TL et al. The diagnosis of low-grade dysplasia in Barrett's esophagus and its implications for disease progression. Am J Gastroenterol 2000; 95: 3383-3387

15 Wo JM, Ray MB, Mayfield-Stokes S et al. Comparison of methylene blue-directed biopsies and conventional biopsies in the detection of intestinal metaplasia and dysplasia in Barrett's esophagus: A preliminary study. Gastrointest Endosc 2001; 54: 294 - 301

${ }^{16}$ Sharma P, Topalovski M, Mayo MS et al. Methylene blue chromoendoscopy for detection of short-segment Barrett's esophagus. Gastrointest Endosc 2001; 54: 289-293

17 Canto MI, Setrakian S, Willis J et al. Methylene blue-directed biopsies improve detection of intestinal metaplasia and dysplasia in Barrett's esophagus. Gastrointest Endosc 2000; 51: 560-568

18 Canto MI, Setrakian S, Petras RE et al. Methylene blue selectively stains intestinal metaplasia in Barrett's esophagus. Gastrointest Endosc 1996; 44: $1-7$

${ }^{19}$ Kiesslich R, Hahn M, Herrmann G et al. Screening for specialized columnar epithelium with methylene blue: chromoendoscopy in patients with Barrett's esophagus and a normal control group. Gastrointest Endosc 2001; 53: 47-52

${ }^{20}$ Dave U, Shousha S, Westaby D. Methylene blue staining: is it really useful in Barrett's esophagus? Gastrointest Endosc 2001; 53: $333-335$

${ }^{21}$ Hackelsberger A, Günther T, Schultze V et al. Intestinal metaplasia at the gastro-oesophageal junction: Helicobacter pylori gastritis or gastro-oesophageal reflux disease? Gut 1998; 43: 17-21

${ }^{22}$ Morales TG, Bhattacharyya A, Johnson C et al. Is Barrett's esophagus associated with intestinal metaplasia of the gastric cardia? Am J Gastroenterol 1997; 92: $1818-1822$

${ }^{23}$ Voutilainen M, Farkkila M, Juhola M et al. Complete and incomplete intestinal metaplasia at the oesophagogastric junction: prevalences and associations with endoscopic erosive oesophagitis and gastritis. Gut 1999; 45: 644-648

24 Voutilainen M, Farkkila M, Juhola M et al. The Central Finland Endoscopy Study Group. Specialized columnar epithelium of the esophagogastric junction: prevalence and associations. Am J Gastroenterol 1999; 94: $913-918$

${ }^{25}$ Meining A, Kiel G, Stolte M. Changes in Helicobacter pylori-induced gastritis in the antrum and corpus during and after 12 months of treatment with ranitidine and lansoprazole in patients with duodenal ulcer disease. Aliment Pharmacol Ther 1998; 12: 735-740

${ }^{26}$ Stolte M, Meining A, Schmitz JM et al. Changes in Helicobacter pyloriinduced gastritis in the antrum and corpus during 12 months of treatment with omeprazole and lansoprazole in patients with gastro-oesophageal reflux disease. Aliment Pharmacol Ther 1998; 12: 247-253

${ }^{27}$ Levine DS, Haggitt RC, Blount PL et al. An endoscopic biopsy protocol can differentiate high-grade dysplasia from early adenocarcinoma in Barrett's esophagus. Gastroenterology 1993; 105: 40-50

${ }^{28}$ Reid BJ, Blount PL, Feng $Z$ et al. Optimizing endoscopic biopsy detection of early cancers in Barrett's high-grade dysplasia. Am J Gastroenterol 2000; 95: 3089-3096

${ }^{29}$ Alexander JA, Jones SM, Smith CJ et al. Usefulness of cytopathology and histology in the evaluation of Barrett's esophagus in a community hospital. Gastrointest Endosc 1997; 46: 318 - 320

${ }^{30}$ Falk GW, Chittajallu R, Goldblum JR et al. Surveillance of patients with Barrett's esophagus for dysplasia and cancer with balloon cytology. Gastroenterology 1997; 112: 1787-1797

${ }^{31}$ Endlicher E, Knuechel R, Hauser T et al. Endoscopic fluorescence detection of low and high grade dysplasia in Barrett's oesophagus using systemic or local 5-aminolaevulinic acid sensitisation. Gut 2001; 48: $314-319$

${ }^{32}$ Reid BJ, Levine DS, Longton G et al. Predictors of progression to cancer in Barrett's esophagus: baseline histology and flow cytometry identify low- and high-risk patient subsets. Am J Gastroenterol 2000; 95: $1669-1676$
${ }^{33}$ Teodori L, Gohde W, Persiani M et al. DNA/protein flow cytometry as a predictive marker of malignancy in dysplasia-free Barrett's esophagus: thirteen-year follow-up study on a cohort of patients. Cytometry 1998; 34: 257-263

${ }^{34}$ Falk GW, Catalano MF, Sivak MV Jr et al. Endosonography in the evaluation of patients with Barrett's esophagus and high-grade dysplasia. Gastrointest Endosc 1994; 40: 207-212

${ }^{35}$ May A, Gunter E, Roth F et al. Accuracy of staging in early oesophageal cancer using high resolution endoscopy and high resolution endosonography: a comparative, prospective, and blinded trial. Gut 2004; 53: $634-40$

${ }^{36}$ Messmann H, Schlottmann K. Role of endoscopy in the staging of esophageal and gastric cancer. Semin Surg Oncol 2001; 20: 78-81

37 Weston AP, Badr AS, Ruth S et al. Prospective multivariate analysis of clinical, endoscopic, and histologicaal factors predicitve of development of Barrett's multifocal high-grade dysplasia or adenocarcinoma. Am J Gastroenterol 1999; 94: 3413-3419

${ }^{38}$ Sampliner RE. Practice guidelines on the diagnosis, surveillance, and therapy of Barrett's esophagus. Am J Gastroenterol 1998; 93: $1028-1032$

${ }^{39}$ Van der Burgh A, Dees J, Hop WC et al. Oesophageal cancer is an uncommon cause of death in patients with Barrett's oesophagus. Gut 1996; 39: 5-8

${ }^{40}$ Iftikhar SY, James PD, Steele RJ et al. Length of Barrett's oesophagus: an important factor in the development of dysplasia and adenocarcinoma. Gut 1992; 33: $1155-1158$

${ }^{41}$ Menke-Pluymers MB, Hop WC, Dees J et al. Risk factors for the development of an adenocarcinoma in columnar-lined (Barrett) esophagus. The Rotterdam Esophageal Tumor Study Group. Cancer 1993; 72: $1155-1158$

${ }^{42}$ Nijhawan PK, Wang KK. Endoscopic mucosal resection for lesions with endoscopic features suggestive of malignancy and high-grade dysplasia within Barrett's esophagus. Gastrointest Endosc 2000; 52: $328-332$

${ }^{43}$ Buttar NS, Wang KK, Sebo TJ et al. Extent of high-grade dysplasia in Barrett's esophagus correlates with risk of adenocarcinoma. Gastroenterology 2001; 120: 1630-1639

${ }^{44}$ Faller G, Berndt R, Borchard F et al. Working Group for Gastroenterological Pathology, German Society for Pathology. Histopathological diagnosis of Barrett's mucosa and associated neoplasias. Results of a consensus conference of the Working Group for "Gastroenterological Pathology of the German Society for Pathology“. Pathologe 2003; 24: $9-14$

${ }^{45}$ Gimenez A, Minguela A, Parrilla P et al. Flow cytometric DNA analysis and p53 protein expression show a good correlation with histologic findings in patients with Barrett's esophagus. Cancer 1998; 83: $641-651$

${ }^{46}$ Kim R, Clarke MR, Melhem MF et al. Expression of p53, PCNA, an C-erbB-2 in Barrett's metaplasia and adenocarcinoma. Dig Dis Sci 1997; 42: $2463-2462$

47 Weston AP, Banerjee SK, Sharma P et al. p53 protein overexpression in low grade dysplasia (LGD) in Barrett's esophagus: immunohistochemical marker predictive of progression. Am J Gastroenterol 2001; 96: $1355-1362$

${ }^{48}$ Van Sandick JW, van Lanschot JJ, Kuiken BW et al. Impact of endoscopic biopsy surveillance of Barrett's oesophagus on pathological stage and clinical outcome of Barrett's carcinoma. Gut 1998; 43: 216-222

${ }^{49}$ Streitz JM Jr, Andrews CW Jr, Ellis FH Jr. Endoscopic surveillance of Barrett's esophagus. Does it help? J Thorac Cardiovasc Surg 1993; 105: $383-387$

${ }^{50}$ Fitzgerald RC, Saeed IT, Khoo D et al. Rigorous surveillance protocol increases detection of curable cancers associated with Barrett's esophagus. Dig Dis Sci 2001; 46: 1892 - 1898

${ }^{51}$ Wright TA, Gray MR, Morris AI et al. Cost effectiveness of detecting Barrett's cancer. Gut 1996; 39: $574-579$

52 Corley DA, Levin TR, Habel LA et al. Surveillance and survival in Barrett's adenocarcinomas: a population-based study. Gastroenterology 2002; 122: $633-640$

${ }^{53}$ Streitz J, Ellis F, Tilden R et al. Endoscopic surveillance of Barrett's esophagus: a cost-effectivness comparison with mammographic surveillance for breast cancer. Am J Gastroenterol 1998; 93: 911 - 915

54 Soni A, Sampliner R, Sonnenberg A. Screening for high-grade dysplasia in gastroesophageal reflux disease: is it cost effective? Am J Gastroenterol 2000; 95: 2086-2093 
${ }^{55}$ Eckhardt VF, Kanzler G, Bernhard G. Life expectancy and cancer risk in patients with Barrett's esophagus: a prospective controlled investigation. Am J Med 2001; 111: 33-37

56 O'Connor J, Falk G, Richter J. The incidence of adenocarcinoma and dysplasia in Barrett's esophagus: report on the Cleveland Clinic Barrett's Esophagus Registry. Am J Gastroenterol 1999; 94: 2037-2042

${ }^{57}$ Shaheen N, Crosby M, Bozymski E et al. Is there a publication bias in the reporting of cancer risk in Barrett's esophagus? Gastroenterology 2000; 119: $333-338$

${ }^{58}$ Jankowski JA, Provenzale D, Moayyedi P. Esophageal adenocarcinoma arising from Barrett's metaplasia has regional variations in the west. Gastroenterology 2002; 122: 588-590

59 Provenzale D, Schmitt C, Wong J. Barrett's esophagus: A new look at surveillance based on emerging estimates of cancer risk. Am J Gastroenterol 1999; 94: 2043 - 2053

${ }^{60}$ Sampliner RE. Practice Parameters Committee of the American College of Gastroenterology. Updated guidelines for the diagnosis, surveillance, and therapy of Barrett's esophagus. Am J Gastroenterol 2002; 97: 1888 - 1895

${ }^{61}$ Weston A, Krmpotich P, Cherian R et al. Prospective long-term endoscopic and histological follow-up of short segment Barrett's esophagus: Comparison with traditional long segment Barrett's esophagus. Am J Gastroenterol 1997; 92: 407-413

${ }^{62}$ Wright TA. High-grade dysplasia in Barrett's oesophagus. Br J Surg 1997; 84: $760-766$

${ }^{63}$ Weston AP, Sharma P, Topalovski M et al. Long-term follow-up of Barrett's high-grade dysplasia. Am J Gastroenterol 2000; 95: 1888 - 1893

${ }^{64}$ Schnell TG, Sontag SJ, Chejfec G et al. Long-term nonsurgical management of Barrett's esophagus with high-grade dysplasia. Gastroenterology 2001; 120: 1607-1619

${ }^{65}$ Falk GW, Rice TW, Goldblum JR et al. Jumbo biopsy forceps protocol still misses unsuspected cancer in Barrett's esophagus with high-grade dysplasia. Gastrointest Endosc 1999; 49: 170-176

${ }^{66}$ Ouatu-Lascar R, Fitzgerald RC, Triadafilopoulos G. Differentiation and proliferation in Barrett's esophagus and the effects of acid suppression. Gastroenterology 1999; 117: 327 - 335

67 Sharma P, Sampliner RE, Camargo E. Normalization of esophageal pH with high-dose proton pump inhibitor therapy does not result in regression of Barrett's esophagus. Am J Gastroenterol 1997; 92: $582-585$

${ }^{68}$ Wilkinson SP, Biddlestone L, Gore S et al. Regression of columnar-lined (Barrett's) oesophagus with omeprazole $40 \mathrm{mg}$ daily: results of 5 years of continuous therapy. Aliment Pharmacol Ther 1999; 13: $1205-1209$

${ }^{69}$ Gore S, Healey CJ, Sutton R et al. Regression of columnar lined (Barrett's) oesophagus with continuous omeprazole therapy. Aliment Pharmacol Ther 1993; 7: 623-628

70 Neumann CS, Iqbal TH, Cooper BT. Long term continuous omeprazole treatment of patients with Barrett's oesophagus. Aliment Pharmacol Ther 1995; 9: 451 - 454

${ }^{71}$ Peters FT, Ganesh S, Kuipers EJ et al. Endoscopic regression of Barrett's oesophagus during omeprazole treatment; a randomised double blind study. Gut 1999; 45: 489-494

${ }^{72}$ Cooper BT, Neumann CS, Cox MA et al. Continuous treatment with omeprazole $20 \mathrm{mg}$ daily for up to 6 years in Barrett's oesophagus. Aliment Pharmacol Ther 1998; 12: $893-897$

${ }^{73}$ Sagar PM, Ackroyd R, Hosie KB et al. Regression and progression of Barrett's oesophagus after antireflux surgery. Br J Surg 1995; 82: $806-810$

${ }^{74}$ McDonald ML, Trastek VF, Allen MS et al. Barrett's esophagus: does an antireflux procedure reduce the need for endoscopic surveillance? J Thorac Cardiovasc Surg 1996; 111: 1135-1138

75 Wang KK. Photodynamic therapy of Barrett's esophagus. Gastrointest Endosc Clin N Am 2000; 10: 409-419

76 Van Laethem JL, Cremer M, Peny MO et al. Eradication of Barrett's mucosa with argon plasma coagulation and acid suppression: immediate and mid term results. Gut 1998; 43: $747-751$
${ }^{77}$ Pereira-Lima JC, Busnello JV, Saul C et al. High power setting argon plasma coagulation for the eradication of Barrett's esophagus. Am J Gastroenterol 2000; 95: 1661 - 1668

${ }^{78}$ Luman W, Lessels AM, Palmer KR. Failure of Nd-YAG photocoagulation therapy as treatment for Barrett's oesophagus - a pilot study. Eur J Gastroenterol Hepatol 1996; 8: 627-630

${ }^{79}$ Wang KK, Sampliner RE. Mucosal ablation therapy of barrett esophagus. Mayo Clin Proc 2001; 76: 433-437

80 Gossner L, May A, Stolte M et al. KTP laser destruction of dysplasia and early cancer in columnar-lined Barrett's esophagus. Gastrointest Endosc 1999; 49: 8-12

${ }^{81}$ Schulz H, Miehlke S, Antos D et al. Ablation of Barrett's epithelium by endoscopic argon plasma coagulation in combination with high-dose omeprazole. Gastrointest Endosc 2000; 51: 659-663

82 Pelligrinin CA, Pohl D. High grade dyplasia in Barrett's esophagus: surveillance or operation? J Gastrointest Surg 2000; 4: 131 - 134

${ }^{83}$ Birkmeyer JD, Siewers AE, Finlayson EV et al. Hospital volume and surgical mortality in the United States. N Engl J Med 2002; 346: $1128-1137$

${ }^{84}$ Barr H, Shepherd NA, Dix A et al. Eradication of high-grade dysplasia in columnar-lined (Barrett's) oesophagus by photodynamic therapy with endogenously generated protoporphyrin IX. Lancet 1996; 348 : $584-585$

${ }^{85}$ Gossner L, Stolte M, Sroka R et al. Photodynamic ablation of high-grade dysplasia and early cancer in Barrett's esophagus by means of 5-aminolevulinic acid. Gastroenterology 1998; 114: 448-455

${ }^{86}$ Ackroyd R, Brown NJ, Davis MF et al. Photodynamic therapy for dysplastic Barrett's oesophagus: a prospective, double blind, randomised, placebo controlled trial. Gut 2000; 47: 612-617

87 Overholt BF, Panjehpour M, Haydek JM. Photodynamic therapy for Barrett's esophagus: follow-up in 100 patients. Gastrointest Endosc 1999; 49: $1-7$

${ }^{88}$ Overholt BF, Panjehpour M, Halberg DL. Photodynamic therapy for Barrett's esophagus with dysplasia and/or early stage carcinoma: long-term results. Gastrointest Endosc 2003; 58: 183- 188

${ }^{89}$ Overholt BF, Lightdale C, Wang C et al. International, multicenter, partially blinded randomised study of the efficacy of PDT using porfirmersodium (POR) for the ablation of high-grade dysplasia (HGD) in Barrett's esophagus (BE): Results of 24-month follow-up. Gastroenterology 2003; 124 (Suppl): 151

${ }^{90}$ Ell C, May A, Gossner L et al. Endoscopic mucosal resection of early cancer and high-grade dysplasia in Barrett's esophagus. Gastroenterology 2000; 118: 670-677

${ }^{91}$ May A, Gossner L, Pech O et al. Local endoscopic therapy for intraepithelial high-grade neoplasia and early adenocarcinoma in Barrett's oesophagus: acute-phase and intermediate results of a new treatment approach. Eur J Gastroenterol Hepatol 2002; 1410: 1085

92 Van Laethem JL, Jagodzinski R, Peny MO et al. Argon plasma coagulation in the treatment of Barrett's high-grade dysplasia and in situ adenocarcinoma. Endoscopy 2001; 33: 257-261

93 Pacifico RJ, Wang KK, Wongkeesong LM et al. Combined endoscopic mucosal resection and photodynamic therapy versus esophagectomy for management of early adenocarcinoma in Barrett's esophagus. Clin Gastroenterol Hepatol 2003; 1: 252 - 257

94 Stein HJ, Feith M, Mueller J et al. Limited resection for early adenocarcinoma in Barrett's esophagus. Ann Surg 2000; 232: 733 - 742

95 Banki F, Mason RJ, DeMeester SR et al. Vagal-sparing esophagectomy: a more physiologic alternative. Ann Surg 2002; 236: 324-335

96 Ell C, May A, Gossner L et al. Kurative endoskopische Therapie früher Adenokarzinome der Speiseröhre. Deutsches Ärzteblatt 2003; 21: A1438 - 1448

97 Sibille A, Lambert R, Souquet JC et al. Long-term survival after photodynamic therapy for esophageal cancer. Gastroenterology 1995; 108: $337-344$ 